\title{
ANALYSIS OF MAGNETO-OPTICAL SPECTRA OF MAGNETIC MATERIALS USING MODIFIED EFFECTIVE MEDIUM THEORY.
}

\author{
Chun-Yeol You and Sung-Chul Shin \\ Department of Physics, Korea Advanced Institute of Science and Technology, Taejon 305-701, Korea
}

\begin{abstract}
We have modified the Bruggeman's effective medium theory(EMT) to study the microstructural dependence of the magneto-optical spectra in magnetic materials having the off-diagonal elements of the dielectric tensor. We have applied this modified EMT to $20-\hat{A}$-thick Co film having island structure and observed that this modified EMT exhibited better match to the experimental data than any other existing theories. KEYWORDS:Magnetooptical Kerr effects, off-diagonal element, effective medium theory.
\end{abstract}

\section{INTRODUCTION}

It is well known that the optical properties of metallic thin films are much different from these of bulk metals, bcause of discontinuity, roughness, and inhomogeneity [1]. Magneto-optical(MO) properties of metallic magnetic thin films therefore depend not only on their composition $[2,3]$ but also on the microstructure $[4-6]$. A few theories have been suggested to explain the dependence of MO spectra on the microstructure of a sample $[7,9,10]$. However, any existing theories failed to quantitatively match the experimental results $[4-6,11]$. We have modified the effective medium theory to explain the deperidence of MO spectra on the microstructure. The Bruggeman [12] effective medium theory (EMT) has been developed for optically isotropic materials having scalar dielectric constants. We have modified the EMT for magnetized rotational ellipsoid having off-diagonal elements of the dielectric tensor. Validity of the modified effective medium theory(MEMT) has been tested to a 20 - $\AA$-thin Co film having island structure. Using the MEMT, behavior of the diagonal and the off-diagonal elements of the dielectric tensor of these 20 - $\AA$-thin Co film could be well explained, and this theory was well matched to the experiemntal data compared to existing theories $[4,5,7]$.

\section{MODOFIED EFFECTIVE MEDIUM THEORY}

The Bruggeman effective medium theory deals a heterogeneous medium, consisting of random cells of more than two kinds of materials, as a homogeneous effective medium having an effective dielectric tensor in a specified wavelength region. In other words, using electromagnetic radiation of much longer wavelength than the size of each composite, we can not distinguish each composite embedded in the effective medium. In the effective medium theory surface ronghness, heterogeneous structure, or composites would be replaced by a suitable effec- tive medium having an effective dielectric constant with a smooth surface parallel to a plane as shown in Fig. 1.

To modify the Bruggeman effective medium theory for a dielectric tensor of a magnetic material having a cubic symmetry, the microstructure of a magnetic material would be modeled by magnetized rotational ellipsoids. The structure is composed of ellipsoids whose dielectric tensors are $\tilde{\epsilon}_{A}$ with probability $f$ and $\tilde{\epsilon}_{B}$ with probability of $1-f$. This ellipsoid is embedded in an effective medium whose dielectric tensor $\ddot{\epsilon}^{\text {eff }}$ can be determined by the effective medium theory. [13]. If a medium $A$ is a magnetic material having a cubic symmetry with $z$ directional magnetization, the dielectric tensor $\tilde{\epsilon}_{A}$ of the medium $A$ is given by [2]

$$
\tilde{\epsilon}_{A}=\left(\begin{array}{ccc}
\epsilon_{x x} & \epsilon_{x y} & 0 \\
-\epsilon_{x y} & \epsilon_{x x} & 0 \\
0 & 0 & \epsilon_{x x}
\end{array}\right)
$$

For a non-magnetic medium $B$, the dielectric tensor $\tilde{\epsilon}_{B}$ is given as follows:

$$
\tilde{\epsilon}_{B}=\epsilon_{0}\left(\begin{array}{lll}
1 & 0 & 0 \\
0 & 1 & 0 \\
0 & 0 & 1
\end{array}\right)
$$

where $\epsilon_{0}$ is a dielectric constant of vacuum. Let the effective dielectric tensor $\hat{\epsilon}^{\text {eff }}$ for an effective medium be expressed as follows:

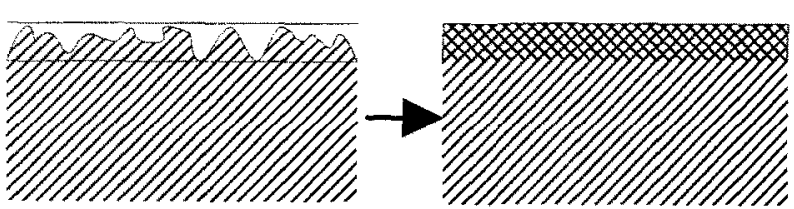
(a) Rough surface layer
(b) Plane paralle: boundary layer

FIG. 1. Replacement of a rough surface layer by an equivalent layer with smooth surface parallel to a plane. 


$$
\hat{\epsilon}^{e f f}=\left(\begin{array}{ccc}
\hat{\epsilon}_{x x} & \hat{\epsilon}_{x y} & 0 \\
-\hat{\epsilon}_{x y} & \hat{\epsilon}_{x x} & 0 \\
0 & 0 & \hat{\epsilon}_{z 2}
\end{array}\right)
$$

If the medium $A$ has no off-diagonal element of the dielectric tensor and an external electric field $\vec{E}_{0}$ is applied along the $x$-direction, the induced polarization vector $\vec{P}_{A}$ of the medium $A$ embedded in the effective medium $\hat{\epsilon}$ eff is given by $[14,15]$

$$
\vec{P}_{A}=\left(\tilde{\epsilon}_{A}-\vec{\epsilon}^{e f f}\right) \cdot \vec{E}^{-},
$$

where the electric field inside the ellipsoid $\vec{E}^{-}$can be expressed as

$$
\hat{\epsilon}^{e f f} \cdot \vec{E}^{-}=\hat{\epsilon}^{e f f} \cdot \vec{E}_{0}-\tilde{L} \cdot \vec{P}_{A} .
$$

In $\mathrm{Eq} .(5), \tilde{L}$ is a depolarization tensor which depends only on the shape of the ellipsoid in the approximation. If we choose the principal axes as the axes of the ellipsoid, $\tilde{L}$ has only the diagonal elements. Moreover, because of the shape dependence of $\tilde{L}, \tilde{L}$ has the same value as the demagnetization factor which is an important magnetic parameter.

It is not unresonable to assume that Eq. (5) is also valid for a magnetic medium having the off-diagonal elements of the dielectric tensor. Using Eq. (4) and (5), the induced polarization $\vec{P}_{A}$ of a magnetic medium $A$ can be expressed as

$$
\vec{P}_{A}=\left(\tilde{\epsilon}_{A}-\hat{\epsilon}^{e f f}\right) \cdot\left(\hat{\epsilon}^{e f f}+\tilde{L} \cdot\left(\tilde{\epsilon}_{A}-\hat{\epsilon}^{e f f}\right)\right)^{-1} \cdot \hat{\epsilon}^{e f f} \cdot \vec{E}_{0} .
$$

If the dielectric tensor of an ellipsoid is $\tilde{\epsilon}_{B}$, expression for the induced polarization vector $\vec{P}_{B}$ is similar to that of $\vec{P}_{A}$, except that $\tilde{\epsilon}_{A}$ is replaced by $\tilde{\epsilon}_{B}$. Since the occupying probabilities, or volume fractions of the medium $A$ and the medium $B$ are $f$ and $1-f$, respectively, the total polarization of the medium is given by $f \vec{P}_{A}+(1-f) \vec{P}_{B}$ [13]. From the definition of the effective medium theory, the total polarization of the effective medium must be zero. So, we can obtain the following relation:

$$
\begin{aligned}
0 & =f\left(\tilde{\epsilon}_{A}-\hat{\epsilon}^{e f f}\right)\left(\hat{\epsilon}^{e f f}+\tilde{L} \cdot\left(\tilde{\epsilon}_{A}-\hat{\epsilon}^{e f f}\right)\right)^{-1} \\
& +(1-f)\left(\tilde{\epsilon}_{B}-\tilde{\epsilon}^{e f f}\right)\left(\left(^{e f f}+\tilde{L} \cdot\left(\tilde{\epsilon}_{B}-\hat{\epsilon}^{e f f}\right)\right)^{-1} .\right.
\end{aligned}
$$

This equation can be rearranged as follows:

$$
\begin{aligned}
0 & =(\tilde{L}-1) \cdot \hat{\epsilon}^{e f f} \cdot \hat{\epsilon}^{e f f} \\
& \left.+\left((f \tilde{L}-\tilde{L}) \cdot \tilde{\epsilon}_{A}+((1-f) \tilde{I}-\tilde{L}) \cdot \tilde{\epsilon}_{B}\right)\right) \cdot \hat{\epsilon}^{e f f} \\
& +\tilde{L} \cdot \tilde{\epsilon}_{A} \cdot \tilde{\epsilon}_{B}
\end{aligned}
$$

where $\tilde{I}$ is a $3 \times 3$ unit tensor. By substituting $L_{x}=$ $L_{y}=L$ and $L_{z}=1-2 L$ in Eq. (8), we can obtain the following relation for the diagonal element $\hat{\epsilon}_{x x}$ in $\tilde{\epsilon}^{\text {eff }}$ to the first-order approximation of $\epsilon_{x y} / \epsilon_{x x}$;

$$
\begin{aligned}
0 & =\epsilon_{x x}^{2}+\left(\frac{f-L}{L-1} \epsilon_{x x}+\frac{1-f-L}{L-1} \epsilon_{0}\right) \hat{\epsilon}_{x x} \\
& +\frac{L}{L-1} \epsilon_{x x} \epsilon_{0} .
\end{aligned}
$$

Eq. (9) is a quadratic equation of $\hat{\epsilon}_{x_{x}}$ with the coefficients dependent on $f, L, \epsilon_{x: c}$, and $\epsilon_{0}$. Eq. (9) is similar to the expression given by the Bruggeman EMT or Xia's theory [10], to the first-order approximation. Using the solution of $\hat{\epsilon}_{x x}$, we can obtain the off-diagonal element $\hat{\epsilon}_{x y}$ of the effective dielectric tensor as follows:

$$
\hat{\epsilon}_{x y}=\frac{\left((f-L) \hat{\epsilon}_{x x}+L \epsilon_{0}\right) \epsilon_{x y}}{2(1-L) \hat{\epsilon}_{x x}+(L-f) \epsilon_{x x}+(f+L-1) \epsilon_{0}} .
$$

It should be emphasized out that Eq. (10) is different from the expressions given by Abe [7] and Xia [10].

\section{COMPARISON OF THE THEORY WITH EXPERIEMTNAL DATA}

Fig. 2 shows the theoretical results obtained by the present and Abe's theories, together with the experimental data of the $20-\AA$-thin Co film having island structure reported in Ref. [4] Here, the solid and dashed lines represent the present and Abe's theories, respectively, and the circles denote the experimental data. To change the dielectric tensor to the conductivity tensor, we have used the following relation:

$$
\epsilon_{i j}=\delta_{i j}+i \frac{4 \pi \sigma_{i j}}{\omega}
$$

In our calculation, we have used the experimental data of the 1000- $\AA$-thick Co measured by Nakajima [5]. Here, we have also ignored the classical size effect.

In Fig. 2, we have used $f$ and $L$ for the fitting parameters. Since the data used were taken from in-situ measurements, we could ignore the effect of oxide layer. If one wants to simultaneously fit the diagonal and off-diagonal elements of the dielectric tensor, one must choose the weighting parameter for the non-linear fitting, because of the difference in the order of magnitude between the diagonal and the off-diagonal elements. However, there is no suitable weighting parameter having physical meaning. The fitting parameters $f, L$, and the merit function $\chi^{2}$ are listed in Table $\mathrm{I}$.

The listed values of $f$ and $L$ are varied for each component. Considering the size distribution of the particles [16], the effects of the mutual interaction of the dipoles, and the mirror image of the substrate [17], the actual dependence of the diagonal and the off-diagonal conductivities on $f$ and $L$ might be changed. Especially, for the mirror image effect of the substrate and the dipole-dipole interaction between the particles [17], the dependence of 

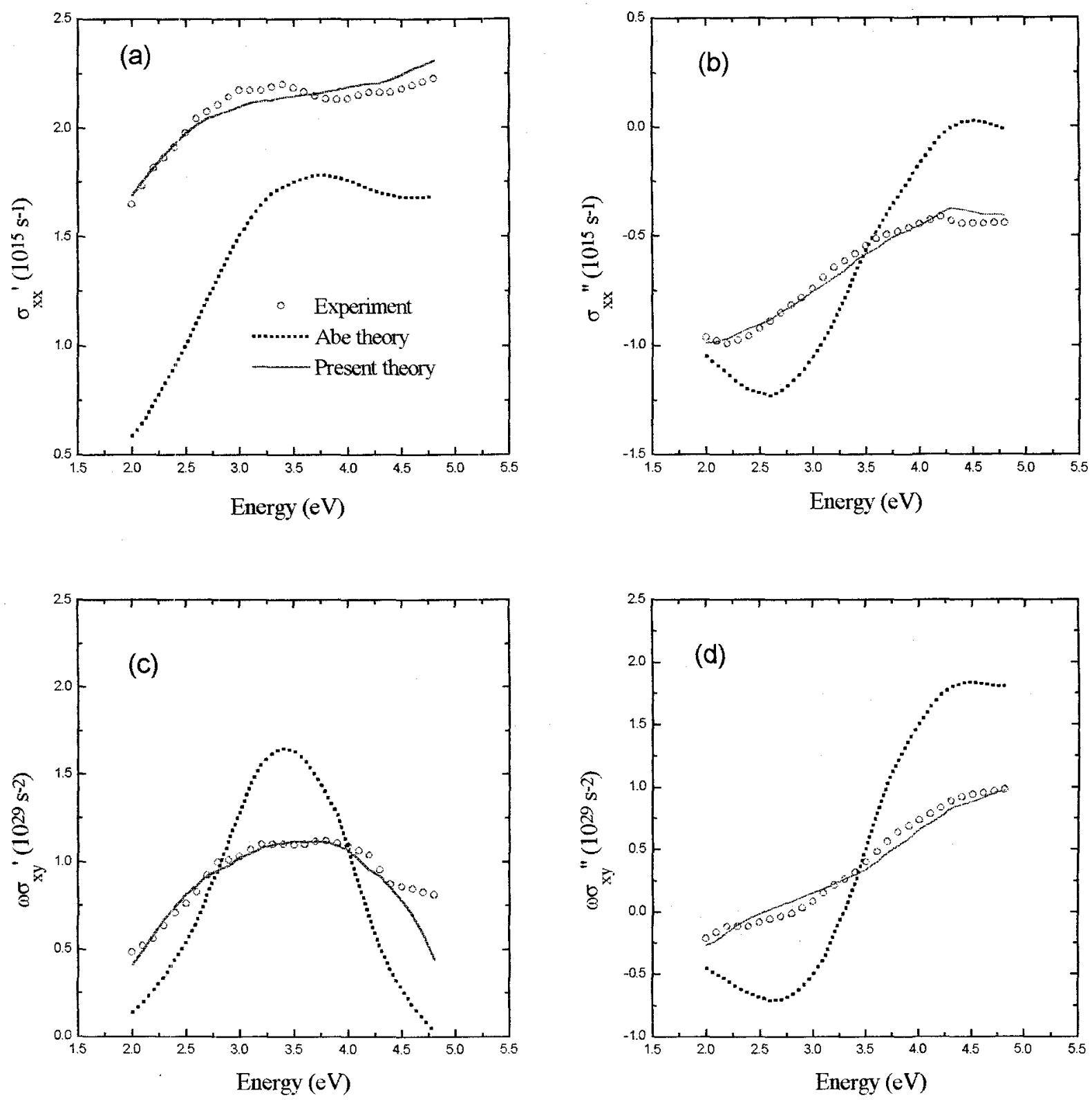

FIG. 2. (a) Real $\left(\sigma_{x x}^{\prime}\right)$, (b) imaginary $\left(\sigma_{x x}^{\prime \prime}\right)$ parts of $\sigma_{x x}$ spectra, (c) real $\left(\omega \sigma_{x y}^{\prime}\right)$ and (d) imaginary $\left(\omega \sigma_{x y}^{\prime \prime}\right)$ parts of $\sigma_{x y}$ spectra of $20-\AA$ Co film. The solid line and dashed lines represent the present and Abe theories, respectively, and the open circles denote the experimental data.

the off-diagonal element might be different from that of the diagonal element.

Using the properties of the depolarization factor, $L_{x}+L_{y}+L_{z}=1$, and assuming that the rotation axis of the ellipsoid to be $z$-axis, then $L_{z}=1-2 L$, since $L_{x}=L_{y}=L$. It is surprising that the values of $L$ in Table I are larger than $\frac{1}{2}$, because $L$ should be less than $\frac{1}{2}$. Marton [18] has pointed out that when one calculates the dielectric constant from the ellipsometric parameters of $\Delta$ and $\Psi$ for the rough surface, the surface has to be dealt as an optically anisotropic medium. The optically anisotropic medium has different dielectric constants for the parallel component, $\epsilon_{\|}$, and the perpendicular component, $\epsilon_{\perp}$, to the electric field. Therefore, using $\Delta$ and 
TABLE $I$. The fitting parameters of $f, L$, and $x^{2}$ for the real and the imaginary parts of $\sigma_{x x}$ and $\omega \sigma_{x y}$.

\begin{tabular}{cccc}
\hline & $f$ & $L$ & $\chi^{2}$ \\
\hline$\sigma_{x x}^{\prime}$ & 0.76 & 0.81 & 0.17 \\
$\sigma_{x x}^{\prime \prime}$ & 0.78 & 0.82 & 0.38 \\
$\omega \sigma_{x y}^{\prime \prime}$ & 0.79 & 0.86 & 0.00049 \\
$\omega \sigma_{x y}^{\prime \prime}$ & 0.83 & 0.92 & 0.00033 \\
\hline \hline
\end{tabular}

$\Psi$, one should calculate both $\epsilon_{\|}$and $\epsilon_{\perp}$ for the rough surface. So, the study of the effective medium theory for an optically anisotropic MO material might be required. Another origin for the abnormality of $L$ could be due to the validity of the Eq. (5) for a magnetic ellipsoid having the off-diagonal element of the dielectric tensor. In such case, the fundamental definition of the depolarization tensor needs a more rigorous mathematical treatment.

\section{CONCLUSIONS}

We have modified the Bruggeman effective medium theory for magnetic materials having the off-diagonal elements of the dielectric tensor. Using the modified effective medium theory, we could well explain the variations of optical and the magneto-optical spectra depending on the changing of the microstructures. We have also well fitted the experimental data for 20 - $\AA$-thin $C o$ film having island structure [4] than any other existing theories $[5,6]$ did. However, the study of an optically anisotropic magnetic medium is required to explain the abnormality of $L$.

\section{ACKNOWLEDGMENTS}

This work was supported by the Ministry of Science and Technology of Korea.

\section{REFERENCES}

[1] D. E. Aspnes: Phys. Rev. B 25, 1358 (1982) and references therein.

[2] K. H. J. Buschow: "Ferromagnetic Materials", Vol 4 edited by E. P. Wohlfarth and K. H. J. Buschow, (NorthHolland, 1988) Ch.5, 493.

[3] W. Reim and J. Schoenes: "Ferromagnetic Materials", Vol 5 edited by E. P. Wohlfarth and K. H. J. Buschow, (North-Holland, 1990) Ch.2, 134.

[4] K. Nakajima and T. Myazaki: J. Magn. Soc. Jpn. 19, Suppl. No.S1, 247 (1995).

[5] K. Nakajima and T. Miyazaki: ISPMM95, Seoul, 899 (1995).

[6] A. Granovoski, E. Ganshina, V. Guschin, A. Vedyaev, B. Dieny, and B. Rodmacq: ISPMM'95. Seoul, 884 (1995).

[7] M. Abe and M. Gomi: Jpn. J. Appl. Phys. 23, 1580 (1984).

[8] D. Stroud: Phys. Rev, B 12,3368 (1075).

[9] P. M. Hui and D. Stroud: Appl. Phys. Lett. 50, 950 (1987).

[10] T. K. Xia, P. M. Hui, and D. Stroud: J. Appl. Phys. 67, $2736(1990)$.

[11] N. A. Yusuf, A. A. Rousan, and H. M. El-Ghanem: J. Appl. Phys. 64, 2781 (1988).

[12] D. A. G. Bruggeman: Ann. Phys. (Leipz.) 24,636 (1935).

[13] G. A. Niklasson, C. G. Granquist, and O. Hunderi: Appl. Opt. 20, 26 (1981).

[14] J. A. Stratton: "Electromagnetic Theory", McGraw Hill, 206 (1941).

[15] L. D. Landan and E. M. Lifshitz: "Electrodynamics of Continuous Media", Pergamon, Oxford (1960).

[16] C. G. Ganviqst and O. Hunderi: Phys. Rev. B 16, 3513 (1977).

[17] S. Yosida, T. Yamaguchi, and A. Kimbara: J. Opt. Soc. Am. 61, 621 (1971).

[18] J. P. Marton and E. C. Chan: J. Appl. Phys. 45, 5008 (1974). 